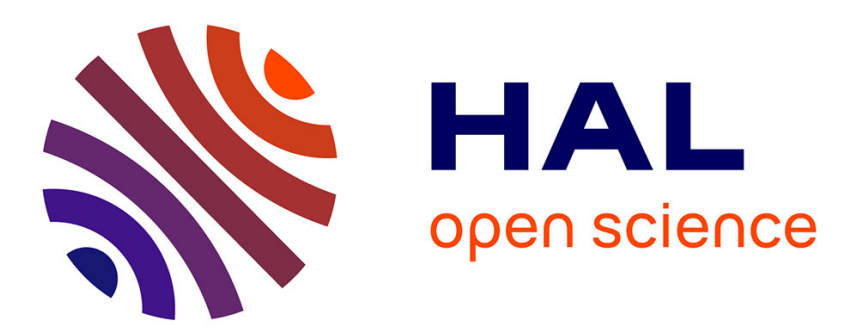

\title{
Its all about connections: hubs and invasion in habitat networks
}

Thibaut Morel-Journel, Claire Rais Assa, Ludovic Mailleret, Elodie Vercken

\section{To cite this version:}

Thibaut Morel-Journel, Claire Rais Assa, Ludovic Mailleret, Elodie Vercken. Its all about connections: hubs and invasion in habitat networks. Ecology Letters, 2019, 22 (2), pp.313-321. 10.1111/ele.13192 . hal-01957100

\author{
HAL Id: hal-01957100 \\ https://hal.inria.fr/hal-01957100
}

Submitted on 17 Dec 2018

HAL is a multi-disciplinary open access archive for the deposit and dissemination of scientific research documents, whether they are published or not. The documents may come from teaching and research institutions in France or abroad, or from public or private research centers.
L'archive ouverte pluridisciplinaire HAL, est destinée au dépôt et à la diffusion de documents scientifiques de niveau recherche, publiés ou non, émanant des établissements d'enseignement et de recherche français ou étrangers, des laboratoires publics ou privés. 
1 Title: It's all about connections: hubs and invasion in habitat networks

2 Authors: Thibaut Morel-Journel ${ }^{1}$, Claire Rais Assa ${ }^{2}$, Ludovic Mailleret $^{2,3}$, Elodie Vercken ${ }^{2}$

3 Affiliations:

$4{ }^{1}$ Earth and Life Institute, Biodiversity Research Centre, Université Catholique de Louvain,

5 Louvain-la-Neuve, Belgium

$6 \quad{ }^{2}$ Université Côte d'Azur, INRA, CNRS, ISA, 06900 Sophia Antipolis, France

$7{ }^{3}$ Université Côte d'Azur, Inria, INRA, CNRS, UPMC Univ. Paris 06, 06900 Sophia Antipolis,

8 France

9 Running title: Hubs and invasion in habitat networks

10 Keywords: Allee effect; connectivity; establishment; hub; individual-based model; invasion;

11 microcosm; network; simulation; spread

12 Type of article: Letters

13 Number of words: 148 (abstract), 4970 (main text)

14 Number of references: 66

15 Number of figures: 2

16 Number of tables: 1

17 Corresponding author: Thibaut Morel-Journel (tel: +3210472759; e-mail:

18 thibaut.morel@uclouvain.be)

19 Statement of authorship: TMJ, LM and EV designed the model and experiments; TMJ and

20 CRA carried out the simulations, experiments and data analyses; all authors participated in the

21 writing of the manuscript and gave their final approval for publication.

22 Data accessibility statement: Should the manuscript be accepted, the data supporting the 23 results and the simulation code will be archived in Dryad and the data DOI will be included at 24 the end of the article. 


\section{Abstract}

2 During the early stages of invasion, the interaction between the features of the invaded

3 landscape, notably its spatial structure, and the internal dynamics of an introduced population,

4 has a crucial impact on establishment and spread. By approximating introduction areas as

5 networks of patches linked by dispersal, we characterized their spatial structure with specific

6 metrics and tested their impact on two essential steps of the invasion process: establishment and

7 spread. By combining simulations with experimental introductions of Trichogramma chilonis

8 (Hymenoptera: Trichogrammatidae) in artificial laboratory microcosms, we demonstrated that

9 spread was hindered by clusters and accelerated by hubs but was also affected by small-

10 population mechanisms prevalent for invasions, such as Allee effects. Establishment was also

11 affected by demographic mechanisms, in interaction with network metrics. These results

12 highlight the importance of considering the demography of invaders as well as the structure of

13 the invaded area to predict the outcome of invasions. 


\section{1}

2

3

4

\section{Introduction}

Managing invasions becomes increasingly costly and decreasingly efficient with the expansion of the invaded area (Simberloff et al. 2013). In invasion biology, focus is placed on the processes occurring during the first generations after the introduction of an exotic species, before its proliferation and spread besides the introduction site. The dispersal abilities of individuals during invasions and the way they evolve over the course of the range expansion have been documented theoretically (Travis et al. 2009; Burton et al. 2010) and experimentally, notably among protists (Fronhofer \& Altermatt 2015) and arthropods (Ochocki \& Miller 2017; Weiss-Lehman et al. 2017). Although the role of individual characteristics on the spread patterns is well known, the way individuals disperse across a landscape also depends on the interaction between individuals and the features of the environment (Calabrese \& Fagan 2004), which can themselves affect the evolution of dispersal (Baguette \& Van Dyck 2007). Therefore, understanding the structure of the invaded landscape is also essential to understand the patterns of colonization observed during the first stages of an invasion.

Networks have gained popularity in the last decades as a method to represent the spatial structure of such landscapes (Urban \& Keitt 2001; Minor \& Urban 2007). They are used to represent habitat patches suitable for the species considered (the vertices of the network) and the way dispersal connects them (the edges of the network). Networks are a powerful tool associated with various metrics and used across a variety of scientific fields, but they are still seldom used in invasion biology. In this field, they are classically used to describe food webs, to study how they are impacted by non-native species or to assess the invasibility of a community based on its trophic structure (Romanuk et al. 2009; Lurgi et al. 2014). A few studies also represent the spatial structure of riverine systems as networks, to study the impact of the network structure on the composition of a community invading a new environment (Seymour \& Altermatt 2014; Alther \& Altermatt 2018). This study aims at using networks in 
another way: to investigate the establishment and spread of the invader, with a special focus on small-population demographic processes.

Although few usages of networks are recorded in invasion biology, other fields provide insight about the impact of the spatial structure of the introduction area on the first stages of an invasion. Notably, several epidemiology studies correlate specific metrics with patterns of spread. A prime example is the combination of networks with models derived from classical Susceptible-Infected (SI) models (Kermack \& McKendrick 1927), e.g. to study the spread of nosocomial infections in hospitals (Ueno \& Masuda 2008) or the transmission of pathogens through grooming among macaques (Romano et al. 2016). These studies usually consider networks made up of hosts (the vertices) connected by social interactions (the edges). Other studies consider larger-scale networks, in which vertices are populations of hosts and edges are host movements between populations (Arino \& Van den Driessche 2006). Such a framework has for example been used to explain the extent of plague during the fourteenth century (Gómez \& Verdú 2017) or to assess epidemic risks in the Japanese airline network (Tanaka et al. 2014). The conceptual similarities between the spread of disease outbreaks and the spread of invading organisms have led studies in invasion biology to use models originating from epidemiological modelling. They notably have been used to study the impact of landscape structure on the risks of invasive spread through forests (Ferrari \& Lookingbill 2009) or across marinas via ballast waters (Floerl et al. 2009). However, these studies fail to consider the internal dynamics of each patch. Moreover, this impact of network structure on invasion remains to be experimentally tested.

Networks are also often used in conservation biology, notably to map the structure of existing metapopulations, in terrestrial (Bunn et al. 2000; Urban \& Keitt 2001), marine (Treml et al. 2008; Almany et al. 2009) and riverine contexts (Schick \& Lindley 2007). Network metrics are notably used to describe the connectivity between sub-populations, to study the 
mean lifetime of metapopulations (e.g. Bode et al. 2008; Drechsler 2009; Kininmonth et al. 2010; Shtilerman \& Stone 2015) or the extinction risks of sub-populations (e.g. Gilarranz \& Bascompte 2012; Peck 2012; Webb \& Padgham 2013). They are also used to identify essential populations to maintain connectivity in a landscape (Bodin \& Saura 2010; Baranyi et al. 2011; Watson et al. 2011). Yet, those studies often focus on the dispersal capabilities of individuals (Bodin \& Saura 2010; Baranyi et al. 2011) or consider local population dynamics only through global colonization and extinction probabilities, without considering explicitly the internal dynamics of the sub-populations (Bode et al. 2008; Gilarranz \& Bascompte 2012; Shtilerman $\&$ Stone 2015). The studies considering the internal dynamics of populations more precisely and its interaction with network features usually concern large, already established populations, and their susceptibility to disturbances, biotic (Mari et al. 2014) or abiotic (Gilarranz et al. 2017). Conversely, the present study focuses on small populations during their growth phase, and on specific associated mechanisms that can interact with the network structure of the landscape. Invaders usually experience multiple demographic bottlenecks, firstly at the initial introduction and subsequently at each colonization event, which can strongly impact the success of an invasion. Mechanisms associated with small population sizes are known to decrease establishment rates if the number of individuals introduced is too low (Simberloff 2009) and to block the spread of invaders to unoccupied patches (Keitt et al. 2001; Johnson et al. 2006). Because of their easily tractable effect on population dynamics, Allee effects are classically considered to account for small population dynamics (Courchamp et al. 2008). However, other mechanisms affect small introduced populations. For instance, they have high risks of going extinct at random, a phenomenon known as demographic stochasticity (Lande et al. 2003). Their probability of not producing any individual able to successfully colonize other patches is also greater, a phenomenon referred to as dispersal stochasticity (Morel-Journel et al. 2016a). 
These demographic mechanisms have been shown to interact with dispersal and impact establishment. Strong emigration notably tends to decrease the establishment rate of introduced populations by decreasing individual density in the introduction site (Kean \& Barlow 2000; Robinet et al. 2008; Morel-Journel et al. 2016b). This study adresses the interplay between the local dynamics of introduced populations and the network structure, during the first stages of an invasion.

Network structure will be characterized using two metrics related to the distribution of edges, i.e. the way patches are connected to one another: clustering and centralization. Clustering indicates the presence of clusters, i.e. groups of vertices well connected to one another (Watts \& Strogatz 1998; Jordán et al. 2003). Clusters have notably been shown to hinder the spread of pathogens (Badham \& Stocker 2010), reduce the extinction risks of already colonized patches (Kininmonth et al. 2010), and buffer the propagation of disturbances across metapopulations (Gilarranz et al. 2017). In the context of invasion biology, they are expected to slow down the spread by "trapping" invaders. Invasions in highly clustered landscape are therefore expected to create fewer, larger populations, which could therefore also be less vulnerable to extinction because of small population mechanisms. Centralization indicates the presence of hubs, i.e. patches concentrating many dispersal flows across the landscape. Hubs are usually central elements in metapopulations, increasing connectivity and the persistence of remote populations otherwise isolated in fragmented landscapes (Watson et al. 2011). They have been shown to increase the speed of infection spread (Ueno \& Masuda 2008; Romano et al. 2016) and to be more likely to generate epidemic outbreaks (Da Silva et al. 2012). In invasion biology, hubs are expected to facilitate the rapid spread of invaders across the 
landscape. Therefore, they may also increase extinction risks of small introduced populations by decreasing the population density locally, especially if the hub is the introduction site itself. We developed an individual-based model to simulate the first generations following introduction, in landscapes whose structure was based on networks with known centralization and clustering levels. In addition, we performed introductions of the parasitoid Trichogramma chilonis, in artificial landscapes, and followed the invasion dynamics during ten generations. We chose the spatial structures of these artificial landscapes among networks used in the simulations to specifically test the impacts of centralization. We performed simulations in the presence of Allee effects or with demographic stochasticity alone, to investigate the interaction between landscape structure and small population mechanisms. T. chilonis does not suffer from Allee effects (Morel-Journel et al. 2016a) but exhibits a strong stochasticity in reproduction and in dispersal. Therefore, it provided a "null biological model" of an introduced population for the experiment. The combination of simulations and experiment is a powerful tool to test hypotheses, and this approach has been at the root of landmark results that have become classics in ecology, e.g. the competitive exclusion principle (Gause 1934), chaotic population dynamics (Cushing et al. 2002), stochasticity in spread rates (Melbourne \& Hastings 2009) or population extinction risks (Drake et al. 2011). Nevertheless, invasion biology - as well as epidemiology and conservation biology, two other fields using spatial network - is heavily dominated by theoretical development and lack experimental validations of classical theoretical predictions, which are essential for the emergence of new hypotheses and the building of a comprehensive ecological theory.

Our results provide the first experimental pieces of evidence of the impacts of landscape structure on the spread of invasive species. The consistency between these results and others in different fields demonstrates that the impacts of centralization and clustering on spreading speed are robust to the ecological context. In addition, our simulations highlight the impact of 
123 Allee effects, not only on the persistence of invasive populations, but also on the colonization

124 speed itself. Another interesting result is that high-density mechanisms, such as over125 competition, can operate even at an early stage and underpin invasion failures depending on the

126 network structure of the landscape. Besides highlighting the importance of landscape structure

127 to predict the outcome of invasions, our study demonstrates its interplay with internal 128 population dynamics. 


\section{Methods}

2 The network structure of the landscapes

3 Using the igraph package (Csardi \& Nepusz 2006) of the R software (R CoreTeam 2018), we

4 generated 112618 networks with a number of vertices $N_{v}=10$ and a number of edges $N_{e}=15$

5 (see Supporting information 1 about the generation and selection of these networks). This set

6 of networks represented all the possible different structure of connected, undirected and

7 unlabeled networks. We computed two metrics to characterize each network: their clustering

8 coefficient $T$ indicating the presence of clusters, and their centralization level $C$ indicating the

9 presence of hubs (see Supporting information 1 about the co mputation of these indices). The

10 value of $C$ is based on two measures of centrality, which encompasses several, conceptually

11 distinct measures, such as the degree, the eigenvector centrality, the betweenness and the

12 closeness. Although all these measures were highly correlated in the set of networks considered

13 (see Supporting information 1), we chose to consider here the closeness and the betweenness.

14 Both were chosen because they carry information about the structure of the paths linking

15 vertices, the closeness accounting for the length of these paths, and the betweenness for their

16 tendency to lie on these paths (Freeman 1978). Because of the correlation between these

17 metrics, we based our measure of centralization on the product between betweenness and

18 closeness, rather than considering them separately. We also identified the most central vertex

19 of a network (called the hub thereafter) based on the product between its betweenness and its

20 closeness. If several vertices shared the highest centrality value, the hub was selected at random

21 among them.

Simulations

24 We developed an individual-based model to simulate invasions in landscape whose spatial 25 structure was described in the previous section (see Supporting information 2 about the 
description of the model). This model was in discrete time, with two successive phases dispersal between patches and local population growth. In this model, individuals had no intrinsic dispersal propensity, and dispersal probability only depends on $d_{i}$ (the degree of the vertex the individual is in) and $p_{e}$ (the dispersal rate of individuals in a vertex with $d_{i}=1$ ). Local population growth was itself divided in two phases. The first concerns mating, affected by $m$, a density-independent mating probability, and $a$, a parameter describing potential mating Allee effects. The second is a reproduction phase, affected by $\alpha$, the intra-specific competition, $\beta$ the fecundity rate of individuals and $s$, the survival rate of juveniles.

Using this model, we simulated 20 time steps after a single initial introduction of 15 individuals in one patch of the landscape, for $p_{e}=0.03, m=0.8, \alpha=0.008, \beta=15$ and $s=0.1$ (see Supporting information 4 for sensitivity analyses of these parameters). The initial population size was chosen so that extinction because of low numbers was possible but not systematic. We simulated $2 \times 2$ invasions for each network in our set, with either $a=0$ (without Allee effects) or $a=2.5$ (with Allee effects), and with an introduction site which was either the hub (the patch corresponding to the most central vertex) or another patch selected at random.

\section{Experiment}

To complement the simulations, we monitored artificial invasions of the egg parasitoid Trichogramma chilonis (Hymenoptera: Trichogrammatidae) in laboratory microcosm landscapes, for ten non-overlapping generations following an initial introduction of 15 individuals (see Supporting information 3 for details about the experimental setup). This model species is especially suited for our experiment, because of its small size (approx. 1mm), its short and regular developmental cycle (consistently 9 days to reach the adult stage), and its ability to parasitize its laboratory host Ephestia kuehniella (Lepidoptera: Pyralidae). The latter allowed us to focus on T.chilonis by suppressing the host's dynamic, and estimate population sizes 
throughout the experiment by counting the parasitized at each generation (see Supporting information 3). Moreover, we could control for impacts of the genetic makeup of the introduced populations by introducing individuals from the same inbred line, founded by a single female and maintained in the laboratory for over 100 generations.

All the microcosm landscapes were made up of 10 patches connected by 15 corridors, and their spatial structure were selected at random in two subsets of the set of networks previously established (see Supporting information 1): the "high-C" and "low-C" landscapes, whose centralization values were respectively in the $10 \%$ highest and $10 \%$ lowest of all the centralization values in the network set. We performed 63 artificial introductions equally distributed across three treatments: (i) in a patch selected at random in a low-C landscape, (ii) in a hub in a high-C landscape, (iii) in another patch than the hub in a high-C landscape. The 63 artificial landscapes were distributed equally across 7 experimental blocks, i.e. 3 replicates of each treatment in each block.

\section{Analysis of the results}

We considered three response variables: the global extinction rate, the introduction site's extinction rate and the colonization speed. The global extinction rate was computed as the proportion of invasions during which all the patches went extinct. The introduction site's extinction rate was computed as the proportion of invasions during which the introduction site went extinct at least once. Both extinction rates followed binomial distributions and were analyzed with logistic regressions. The colonization speed was computed as the ratio between the maximal number of patches colonized and the number of generations to reach this maximal extent. Considering the maximum extent allowed us to differentiate failed establishments from the start from "boom-and-bust" invasions. The colonization speed had a continuous and positive distribution clustered towards low values and was therefore analyzed with a gamma regression. 
we considered a fourth response variable for the experiment: the size reached by a local population at the generation preceding its extinction. These values were distributed as an overdispersed Poisson distribution, that were analyzed using a negative binomial regression. We used AIC comparisons to assess the respective importance of $C$ and $T$ as explanatory variables in the simulations. We considered a model including both variables ( $C T$ model), one

81 with only $C$ ( $C$ model) and one with only $\mathrm{T}$ ( $T$ model). We computed $\Delta_{C}$ as the difference 82 between the AIC of the $C T$ model and the $T$ model, and $\Delta_{T}$ as the difference between the AIC 83 of the $C T$ model and the $C$ model. We considered that values of $\Delta_{T}$ or $\Delta_{C}$ greater than 10 84 indicated that the support for the $C T$ model was unequivocally higher than for the other model 85 (Burnham \& Anderson 2003). To analyze the experimental results, we used generalized linear 86 mixed models, to account for potential differences created by the experimental blocks. The 87 experimental block was treated as a crossed random effect, as the treatments were distributed 88 equally across all blocks. 


\section{Results}

\section{Simulations}

3 The global extinction rate was always negligible in the simulations when $a=0$ (no Allee effect),

4 regardless of the introduction site or the landscape characteristics (Figure 1A, B). It was on 5 average higher when $a=2.5$ but was independent from clustering whether the introduction was 6 performed in the hub $\left(\Delta_{T}=-1.02\right)$ or elsewhere in the landscape $\left(\Delta_{T}=-1.87\right)$. The 7 centralization level had an impact, but only when $a=2.5$ and for an introduction in the hub $8 \quad\left(\Delta_{C}=602.37\right)$. In this case, the global extinction rate increased with $C$ (Figure 1A).

The introduction site's extinction rate was also overall higher when $a=2.5$ than when $a=0$ but was impacted by centralization regardless of Allee effects when individuals were

11 introduced in the hub (Table 1). In this case, centralization increased the extinction rate, with a 12 large majority of introduction sites going extinct at high $C$ levels (Figure 1C). The impact of clustering appeared only for introductions in the hub and $a=2.5\left(\Delta_{T}=9.70\right)$. In this case, it decreased slightly the extinction rate of the introduction site (Figure 1D).

Centralization had a substantial positive impact on colonization speeds when individuals were introduced in the hub itself (Figure 1E), whether $\mathrm{a}=0\left(\Delta_{C}=1578.84\right)$ or $a=2.5$ $\left(\Delta_{C}=3250.45\right)$. Its positive impact was weaker for introductions outside of the hub, although it was still significant when $a=0\left(\Delta_{C}=130.50\right.$, Figure $\left.1 \mathrm{E}\right)$. Clustering markedly decreased the colonization speeds when $a=0$, when introductions were performed in the hub $\left(\Delta_{T}=865.15\right)$ or elsewhere $\left(\Delta_{T}=648.80\right)$. Its impact was however limited when $a=2.5$ for introductions in the hub $\left(\Delta_{T}=29.20\right)$ and negligible for introductions outside of the hub $\left(\Delta_{T}=-0.58\right)$. Overall, the colonization speeds were higher when $a=0$ compared to $a=2.5$.

The sensitivity analyses performed on the parameters of the model (see Supporting information 4) showed that, although the parameters could have an impact on the values of the response variables (the global extinction rate, the introduction site's extinction rate or the 
colonization speed), they did not affect qualitatively the relationships between centralization or clustering, and these response variables.

\section{Experiment}

We were not able to evidence differences between the three experimental treatments concerning the extinction rates (Figure 2), neither at the level of the whole landscape (Likelihood Ratio

32 Test, $\mathrm{df}=2 ; \mathrm{p}=0.344)$ nor at the level of the introduction site (Likelihood Ratio Test, $\mathrm{df}=2$; $\mathrm{p}=0.747)$. However, the sizes of the local populations going extinct during the invasions of high-C landscapes after an introduction outside of the hub were significantly higher than those in low-C landscapes (Wald test; $\mathrm{z}=2.325, \mathrm{p}=0.021$ ) and those in high-C landscapes with introductions in the hub (Wald test; $\mathrm{z}=2.951, \mathrm{p}=0.004$ ).

The colonization speeds recorded during the experiment were overall low (way less than one patch per generation on average) but they were significantly higher in the high-C landscapes compared to the low-C landscapes (Wald test; $\mathrm{z}=2.980, \mathrm{p}=0.0014$ ), but only when the hub 


\section{Discussion}

2 Impacts of landscape structure on spread

3 Both the simulations and the experiments evidenced an impact of landscape structure on spread.

4 The colonization of new patches by invaders was slowed down by the presence of clusters and

5 accelerated by the presence of hubs. The effect of centralization was dominant when individuals

6 were initially introduced in the hub itself, although it was still significant when the introduction

7 site was chosen at random in the simulations. Our results are consistent with other studies in other fields, and thus confirm the effect of the network structure itself on spread, regardless of context.

Centrality was repeatedly shown to facilitate the transmission of infections (e.g.

11 Christley et al. 2005; Ueno \& Masuda 2008; Da Silva et al. 2012; Romano et al. 2016).

12 Epidemics starting in hubs were shown to reach greater sizes, thus underlining the influence of the hub at the start of spread (Da Silva et al. 2012), which we confirm in the context of invasions simulations and experiments. Other studies showed that the most central agents also have higher chances of being infected (Christley et al. 2005; Romano et al. 2016). In studies in epidemiology considering networks of populations, the most central vertices are susceptible to re-infections, even after the initial epidemic outbreak. This phenomenon, likely behind some of the greatest epidemics (e.g. Gómez \& Verdú 2017), also underpins major challenges for the control of invasive populations: the most central patches do not only increase the speed of invasive spread but are also more likely to be invaded.

Our simulation results concerning clustering are also consistent with epidemiological studies showing its role in limiting the speed and extent of infections (Keeling 2005; Miller 2009; Badham \& Stocker 2010). Moreover, Gilarranz et al. (2017) provide experimental evidence that modularity also prevents spread in a similar context: the propagation of disturbances. Modularity, which is particularly used in trophic (Dormann \& Strauss 2014; 
Beckett 2016) and genetic networks (Fletcher Jr et al. 2013; Peterman et al. 2016), describes the belonging of vertices to modules, i.e. subparts of the network highly connected (Newman \& Girvan 2004). In a metapopulation context, Gilarranz et al. (2017) show that disturbances occurring in one module mostly affect other populations in this module, similarly to the way clusters "trap" introduced individuals in our simulation results.

Although they did not dramatically change the impacts of centralization or clustering, Allee effects reduced the colonization speed in the simulations. This result is consistent with the theoretical predictions of Keitt et al. (2001), which suggest that Allee effects can act as a supplementary hurdle to colonization, preventing the small populations at the margin of the invaded area to produce enough dispersing individuals to successfully colonizing new patches. This impact of population dynamics on colonization speed echoes theoretical results on the variation of speed rates with density-dependent growth (Sullivan et al. 2017) and on pushed invasion (Lewis \& Kareiva 1993; Roques et al. 2012). The low colonization speeds observed during our microcosm invasions indicated that colonization failures also occurred during the experiment, creating a "pushed-like" invasion pattern likely caused by dispersal stochasticity, as evidenced in Morel-Journel et al. (2016a) in the same experimental system.

\section{Impacts of landscape structure on establishment}

In addition to affecting the spread of invaders, the network structure of the introduction area also impacted the dynamics of the introduced populations. While the results concerning spread suggest that the same mechanisms were at play in the experiment and the simulations, extinction appears to be underpinned by different causes. In the simulations, the centralization of the landscape increased the introduction site's extinction rate when individuals were introduced in the hub. This result highlights a tradeoff between colonization and local persistence at the beginning of invasions, which is consistent with previous works in invasion 
biology. Theoretical (Lewis \& Kareiva 1993; Kanarek et al. 2013) and empirical (Robinet et al. 2008; Vercken et al. 2011) studies underline the negative impact of dispersal soon after the introduction on the persistence of introduced populations. However, the mechanism invoked by these studies to explain these extinctions is the Allee effect, while our simulation results show that the extinction of the introduction site can occur because of demographic stochasticity alone.

We also observed local extinctions during the experiment, although they occurred regardless of the treatment considered. If some extinctions occurring at small population sizes can be attributed to demographic stochasticity, other populations went extinct while they were close to the carrying capacity, which would rather suggest that they suffered from overcompetition. This would be consistent with the biology of T. chilonis, which can be subject to strong over-competition through superparasitism (Suzuki et al. 1984). This phenomenon occurred most often for introductions outside of the hub in high-C landscapes, i.e. in poorly connected patches. Because of their low dispersal rate, individuals mostly remained in the isolated introduction site, where they rapidly suffered from superparasitism and eventually went extinct.

Complete establishment failures did not only require the extinction of the introduction site, but also that individuals fail to colonize other patches in the landscape. While we recorded extinctions of the introduction site regardless of Allee effects in the simulations, most of them did no lead to global extinctions for $a=0$. Interestingly, the most important impact of Allee effects on establishment was to prevent the colonization of new patches in the landscape. During the experiment, colonization failures resulted from an extinction of the introduction site, 
either because of demographic stochasticity or over-competition, combined with colonization failures because of low dispersal rate and dispersal stochasticity.

\section{Impact of the size of the landscape}

The number of vertices of the landscapes used in this study $\left(N_{v}=10\right)$ was not only smaller than the values used for epidemiology studies, but also smaller than the sizes used for network describing entire landscapes. As we aimed at describing invasions dynamics just after the introduction, we created landscapes corresponding to the direct surroundings of the introduction site only. To test the validity of the results presented in larger landscapes, we performed additional simulations, with networks with a larger number of edges $\left(N_{e}\right.$ varying between 20 and 40) and with a larger number of vertices $\left(N_{v}\right.$ varying between 20 and 100) (see Supporting information 5). The results indicate that the effects of clustering were robust to variations in the number of vertices, while the effects of centralization became weaker, although they remained qualitatively the same. The effects of these metrics were also robust to variations in the number of edges, up to a point. For high values of $N_{e}$, such network-level metrics become irrelevant, as almost every patch can be considered a hub and belonging to a cluster.

\section{Impact of dispersal behavior of the individuals}

In our model, the dispersal of individuals was treated as a diffusion process. Hence, individuals had no intrinsic dispersal propensity, and dispersed only depending on the way the introduction site was connected to the rest of the landscape. Previous results by Morel-Journel et al. (2016b) demonstrated that this approximation is valid to describe the dispersal of $T$. chilonis across our experimental system. Yet, we completed our results by considering emigration rates dependent on the choice of individuals, rather than the structure of the landscape (see Supporting information 6). Dispersal choice based on environmental quality would not have been relevant 
to our study, as the sameness of habitat quality across all patches of the landscape is a central aspect of the study. We rather focused on the impact of the presence of conspecifics, i.e. densitydependent dispersal. The results we obtained were qualitatively similar to those previously obtained on spread and on the extinction rate of the introduction site, with little impact of the strength of the density-dependence of dispersal. These additional simulations therefore suggest that the patterns observed in our simulations are robust to variations in the dispersal propensity of individuals. However, we did not test the potential role of the patch quality on this propensity, as all the patches in all our landscapes were considered having the same quality.

Besides these external factors, the dispersal behavior of individuals can also depend on intrinsic differences between individuals. During spread, dispersal is not only impacted by the genetic background of the introduced individuals, but also affects the spatial distribution of genotypes across space. This feedback loop was not considered in our model because all the individuals were considered identical, but such evolution during range expansion has been documented experimentally (Fronhofer \& Altermatt 2015; Ochocki \& Miller 2017; WeissLehman et al. 2017). Yet, we expected the impact of the genetic background of individuals or potential variations between populations to be minimal during our experiment, because of the very low genetic variability in the populations initially introduced (15 individuals from the same inbred line).

\section{Conclusion}

Predicting the fate of introduced species remains a central objective of invasion biology. This study is a first demonstration of the use of network theory in this context to characterize the structure of landscapes and predict their invasibility. We built upon previous results in epidemiology or conservation to investigate the robustness of the influence of network structure on spread patterns. In addition, our study highlights interactions between the two network 
123 metrics studied - centralization and clustering - and small population dynamics characteristic

124 of early stages of invasion. Among the involved small population mechanisms, Allee effects

125 often provide a simple and elegant way to describe the positive density dependence occurring

126 in small introduced populations, but they lack generality and empirical support (Kramer et al.

127 2009; Gregory et al. 2010). Our results demonstrate that demographic and dispersal

128 stochasticities can create similar patterns, by bringing small, well connected populations to

129 extinction, or by preventing colonization. Moreover, negative density-dependence and

130 competitive interactions can also interact with landscape structure and affect the outcome of

131 potential invasions. 


\section{Acknowledgements}

2 We gratefully acknowledge the support of the Department Santé des Plantes et Environnement

3 from the INRA. This research was supported in part by the European Commission through the

47 th Framework Program (PURE project, contract number 265865). This work is a part of the

5 thesis of TMJ, financed by the University Nice Sophia Antipolis. The funders had no role in

6 study design, data collection and analysis, decision to publish, or preparation of the manuscript. 


\section{References}

Alther, R. \& Altermatt, F. (2018). Fluvial network topology shapes communities of native and non-native amphipods. Ecosphere, 9.

Arino, J. \& Van den Driessche, P. (2006). Disease spread in metapopulations. Nonlinear dynamics and evolution equations, 48, 1-13.

Badham, J. \& Stocker, R. (2010). The impact of network clustering and assortativity on epidemic behaviour. Theoretical population biology, 77, 71-75.

Baguette, M. \& Van Dyck, H. (2007). Landscape connectivity and animal behavior: functional grain as a key determinant for dispersal. Landscape ecology, 22, 1117-1129.

Baranyi, G., Saura, S., Podani, J. \& Jordán, F. (2011). Contribution of habitat patches to network connectivity: redundancy and uniqueness of topological indices. Ecological Indicators, 11, 1301-1310.

Beckett, S.J. (2016). Improved community detection in weighted bipartite networks. Royal Society open science, 3, 140536.

Bode, M., Burrage, K. \& Possingham, H.P. (2008). Using complex network metrics to predict the persistence of metapopulations with asymmetric connectivity patterns. ecological modelling, 214, 201-209.

Bodin, Ö. \& Saura, S. (2010). Ranking individual habitat patches as connectivity providers: integrating network analysis and patch removal experiments. Ecological Modelling, 221, 2393-2405.

Burnham, K.P. \& Anderson, D.R. (2003). Model selection and multimodel inference: a practical information-theoretic approach. Springer Science \& Business Media.

Christley, R., Pinchbeck, G., Bowers, R., Clancy, D., French, N., Bennett, R., et al. (2005). Infection in Social Networks: Using Network Analysis to Identify High-Risk Individuals. Am J Epidemiol, 162, 1024-1031.

Courchamp, F., Berec, L. \& Gascoigne, J. (2008). Allee effects in ecology and conservation. Oxford University Press.

Csardi, G. \& Nepusz, T. (2006). The igraph software package for complex network research. InterJournal, Complex Systems, 1695, 1-9.

Cushing, J.M., Costantino, R.F., Dennis, B., Desharnais, R. \& Henson, S.M. (2002). Chaos in ecology: experimental nonlinear dynamics. Elsevier.

Da Silva, R.A.P., Viana, M.P. \& da Fontoura Costa, L. (2012). Predicting epidemic outbreak from individual features of the spreaders. Journal of Statistical Mechanics: Theory and Experiment, 2012, P07005.

Dormann, C.F. \& Strauss, R. (2014). A method for detecting modules in quantitative bipartite networks. Methods in Ecology and Evolution, 5, 90-98.

Drake, J.M., Shapiro, J. \& Griffen, B.D. (2011). Experimental demonstration of a two-phase population extinction hazard. Journal of the Royal Society Interface, 8, 1472-1479.

Drechsler, M. (2009). Predicting metapopulation lifetime from macroscopic network properties. Mathematical biosciences, 218, 59-71.

Ferrari, J.R. \& Lookingbill, T.R. (2009). Initial conditions and their effect on invasion velocity across heterogeneous landscapes. Biological Invasions, 11, 1247-1258.

Fletcher Jr, R.J., Revell, A., Reichert, B.E., Kitchens, W.M., Dixon, J.D. \& Austin, J.D. (2013). Network modularity reveals critical scales for connectivity in ecology and evolution. Nature communications, 4, 2572.

Floerl, O., Inglis, G., Dey, K. \& Smith, A. (2009). The importance of transport hubs in steppingstone invasions. Journal of Applied Ecology, 46, 37-45.

Freeman, L.C. (1978). Centrality in social networks conceptual clarification. Social networks, $1,215-239$. 
Fronhofer, E.A. \& Altermatt, F. (2015). Eco-evolutionary feedbacks during experimental range expansions. Nature communications, 6, 6844.

Gause, G.F. (1934). The Struggle for Existence. Wiliams \& Wilkins, Baltimore.

Gilarranz, L.J. \& Bascompte, J. (2012). Spatial network structure and metapopulation persistence. Journal of Theoretical Biology, 297, 11-16.

Gilarranz, L.J., Rayfield, B., Liñán-Cembrano, G., Bascompte, J. \& Gonzalez, A. (2017). Effects of network modularity on the spread of perturbation impact in experimental metapopulations. Science, 357, 199-201.

Gómez, J.M. \& Verdú, M. (2017). Network theory may explain the vulnerability of medieval human settlements to the Black Death pandemic. Scientific reports, 7, 43467.

Gregory, S.D., Bradshaw, C.J., Brook, B.W. \& Courchamp, F. (2010). Limited evidence for the demographic Allee effect from numerous species across taxa. Ecology, 91, 2151-2161.

Johnson, D.M., Liebhold, A.M., Tobin, P.C. \& Bjørnstad, O.N. (2006). Allee effects and pulsed invasion by the gypsy moth. Nature, 444,361 .

Jordán, F., Báldi, A., Orci, K.-M., Racz, I. \& Varga, Z. (2003). Characterizing the importance of habitat patches and corridors in maintaining the landscape connectivity of a Pholidoptera transsylvanica (Orthoptera) metapopulation. Landscape Ecology, 18, 8392.

Kanarek, A.R., Webb, C.T., Barfield, M. \& Holt, R.D. (2013). Allee effects, aggregation, and invasion success. Theoretical ecology, 6, 153-164.

Kean, J. \& Barlow, N. (2000). Effects of dispersal on local population increase. Ecology letters, 3, 479-482.

Keeling, M. (2005). The implications of network structure for epidemic dynamics. Theoretical population biology, 67, 1-8.

Keitt, T.H., Lewis, M.A. \& Holt, R.D. (2001). Allee effects, invasion pinning, and species' borders. The American Naturalist, 157, 203-216.

Kermack, W.O. \& McKendrick, A.G. (1927). A Contribution to the Mathematical Theory of Epidemics. Proceedings of the Royal Society of London A: Mathematical, Physical and Engineering Sciences, 115, 700-721.

Kininmonth, S., Drechsler, M., Johst, K. \& Possingham, H. (2010). Metapopulation mean life time within complex networks. Marine Ecology Progress Series, 417, 139-149.

Kramer, A.M., Dennis, B., Liebhold, A.M. \& Drake, J.M. (2009). The evidence for Allee effects. Population Ecology, 51, 341.

Lande, R., Engen, S. \& Saether, B. (2003). Stochastic population models in ecology and conservation. Oxford University Press, Oxford.

Lewis, M. \& Kareiva, P. (1993). Allee dynamics and the spread of invading organisms. Theoretical Population Biology, 43, 141-158.

Lurgi, M., Galiana, N., López, B.C., Joppa, L.N. \& Montoya, J.M. (2014). Network complexity and species traits mediate the effects of biological invasions on dynamic food webs. Frontiers in Ecology and Evolution, 2, 36.

Melbourne, B.A. \& Hastings, A. (2009). Highly variable spread rates in replicated biological invasions: fundamental limits to predictability. Science, 325, 1536-1539.

Miller, J.C. (2009). Spread of infectious disease through clustered populations. Journal of the Royal Society Interface, rsif-2008.

Morel-Journel, T., Girod, P., Mailleret, L., Auguste, A., Blin, A. \& Vercken, E. (2016a). The highs and lows of dispersal: how connectivity and initial population size jointly shape establishment dynamics in discrete landscapes. Oikos, 125, 769-777.

Morel-Journel, T., Piponiot, C., Vercken, E. \& Mailleret, L. (2016b). Evidence for an optimal level of connectivity for establishment and colonization. Biol Lett, 12. 
Newman, M.E. \& Girvan, M. (2004). Finding and evaluating community structure in networks. Physical review E, 69, 026113.

Ochocki, B.M. \& Miller, T.E. (2017). Rapid evolution of dispersal ability makes biological invasions faster and more variable. Nature communications, 8, 14315.

Peck, S.L. (2012). Networks of habitat patches in tsetse fly control: Implications of metapopulation structure on assessing local extinction probabilities. Ecological modelling, 246, 99-102.

Peterman, W.E., Ousterhout, B.H., Anderson, T.L., Drake, D.L., Semlitsch, R.D. \& Eggert, L.S. (2016). Assessing modularity in genetic networks to manage spatially structured metapopulations. Ecosphere, 7, e01231.

R CoreTeam. (2018). $R$ : A language and environment for statistical computing. $R$ Foundation for Statistical Computing, Vienna, Austria. 2018.

Robinet, C., Lance, D.R., Thorpe, K.W., Onufrieva, K.S., Tobin, P.C. \& Liebhold, A.M. (2008). Dispersion in time and space affect mating success and Allee effects in invading gypsy moth populations. Journal of Animal Ecology, 77, 966-973.

Romano, V., Duboscq, J., Sarabian, C., Thomas, E., Sueur, C. \& MacIntosh, A.J. (2016). Modeling infection transmission in primate networks to predict centrality-based risk. American journal of primatology, 78, 767-779.

Romanuk, T.N., Zhou, Y., Brose, U., Berlow, E.L., Williams, R.J. \& Martinez, N.D. (2009). Predicting invasion success in complex ecological networks. Philosophical Transactions of the Royal Society B: Biological Sciences, 364, 1743-1754.

Roques, L., Garnier, J., Hamel, F. \& Klein, E.K. (2012). Allee effect promotes diversity in traveling waves of colonization. Proceedings of the National Academy of Sciences, 109, 8828-8833.

Schick, R.S. \& Lindley, S.T. (2007). Directed connectivity among fish populations in a riverine network. Journal of applied ecology, 44, 1116-1126.

Seymour, M. \& Altermatt, F. (2014). Active colonization dynamics and diversity patterns are influenced by dendritic network connectivity and species interactions. Ecology and evolution, 4, 1243-1254.

Shtilerman, E. \& Stone, L. (2015). The effects of connectivity on metapopulation persistence: network symmetry and degree correlations. Proc. R. Soc. B, 282, 20150203.

Simberloff, D. (2009). The role of propagule pressure in biological invasions. Annual Review of Ecology, Evolution, and Systematics, 40, 81-102.

Simberloff, D., Martin, J.-L., Genovesi, P., Maris, V., Wardle, D.A., Aronson, J., et al. (2013). Impacts of biological invasions: what's what and the way forward. Trends in ecology \& evolution, 28, 58-66.

Sullivan, L.L., Li, B., Miller, T.E., Neubert, M.G. \& Shaw, A.K. (2017). Density dependence in demography and dispersal generates fluctuating invasion speeds. Proceedings of the National Academy of Sciences, 114, 5053-5058.

Suzuki, Y., Tsuji, H. \& Sasakawa, M. (1984). Sex allocation and effects of superparasitism on secondary sex ratios in the gregarious parasitoid, Trichogramma chilonis (Hymenoptera: Trichogrammatidae). Animal Behaviour, 32, 478-484.

Tanaka, G., Urabe, C. \& Aihara, K. (2014). Random and targeted interventions for epidemic control in metapopulation models. Scientific reports, 4, 5522.

Ueno, T. \& Masuda, N. (2008). Controlling nosocomial infection based on structure of hospital social networks. Journal of Theoretical Biology, 254, 655-666.

Vercken, E., Kramer, A., Tobin, P. \& Drake, J. (2011). Critical patch size generated by Allee effect in gypsy moth, Lymantria dispar (L.). Ecology letters, 14, 179-186. 
147 Watson, J.R., Siegel, D.A., Kendall, B.E., Mitarai, S., Rassweiller, A. \& Gaines, S.D. (2011). 148 Identifying critical regions in small-world marine metapopulations. Proceedings of the $149 \quad$ National Academy of Sciences, 108, E907-E913.

150 Watts, D.J. \& Strogatz, S.H. (1998). Collective dynamics of "small-world"networks. nature, $151393,440$.

Webb, J.A. \& Padgham, M. (2013). How does network structure and complexity in river 153 systems affect population abundance and persistence? Limnologica-Ecology and Management of Inland Waters, 43, 399-403.

Weiss-Lehman, C., Hufbauer, R.A. \& Melbourne, B.A. (2017). Rapid trait evolution drives increased speed and variance in experimental range expansions. Nature communications, 8, 14303. 


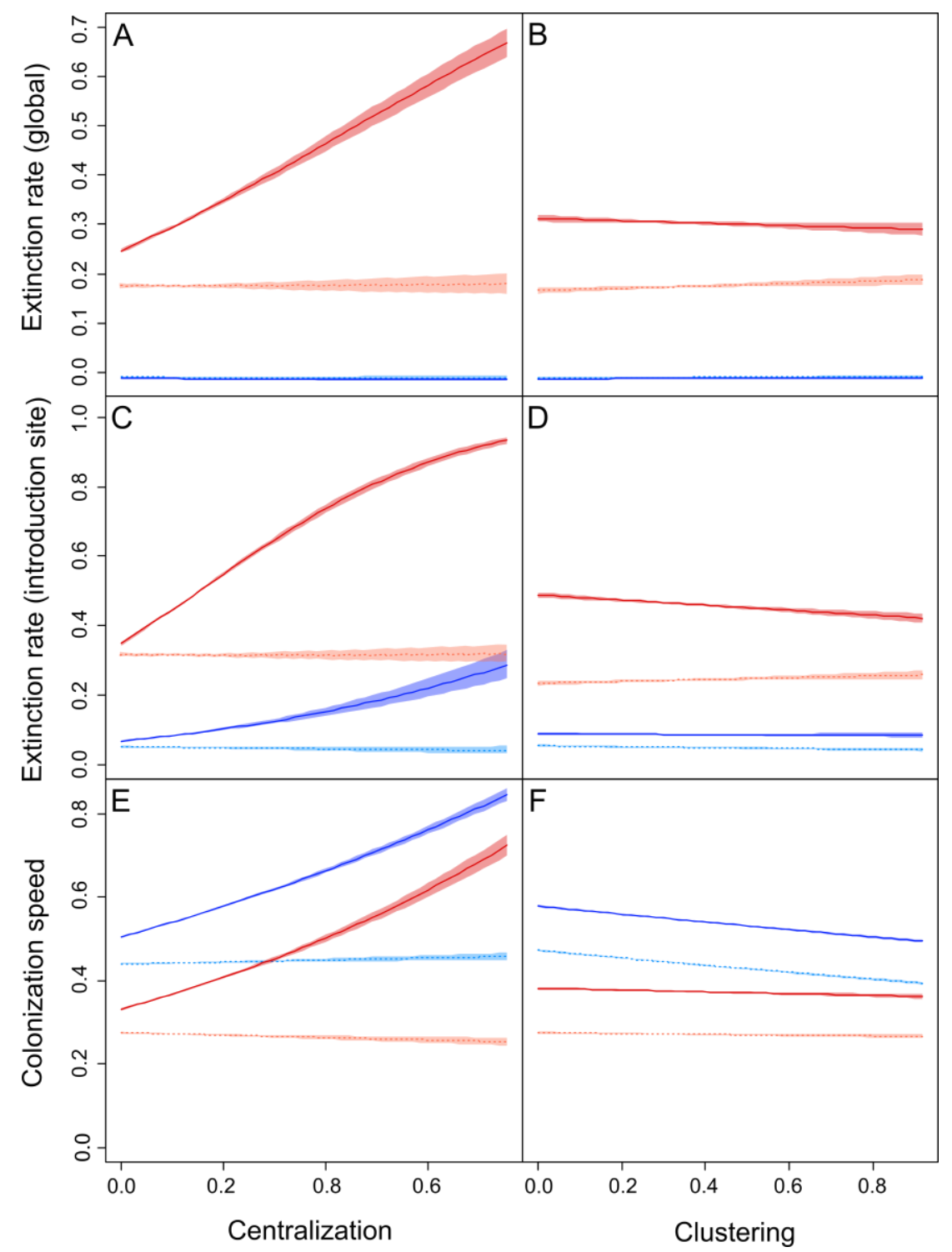

3 Figure 1: Estimated impacts of centralization (A, C, E) and modularity (B, D, F) on the global

4 extinction rate (A, B), introduction site's extinction rate (D, E) and colonization speeds $(\mathrm{E}, \mathrm{F})$,

5 with their 95\% confidence intervals), for $a=0$ (blue) and $a=2.5$ (red) and for an introduction 6 in the hub (full lines, dark) and outside the hub (dashed lines, light). 

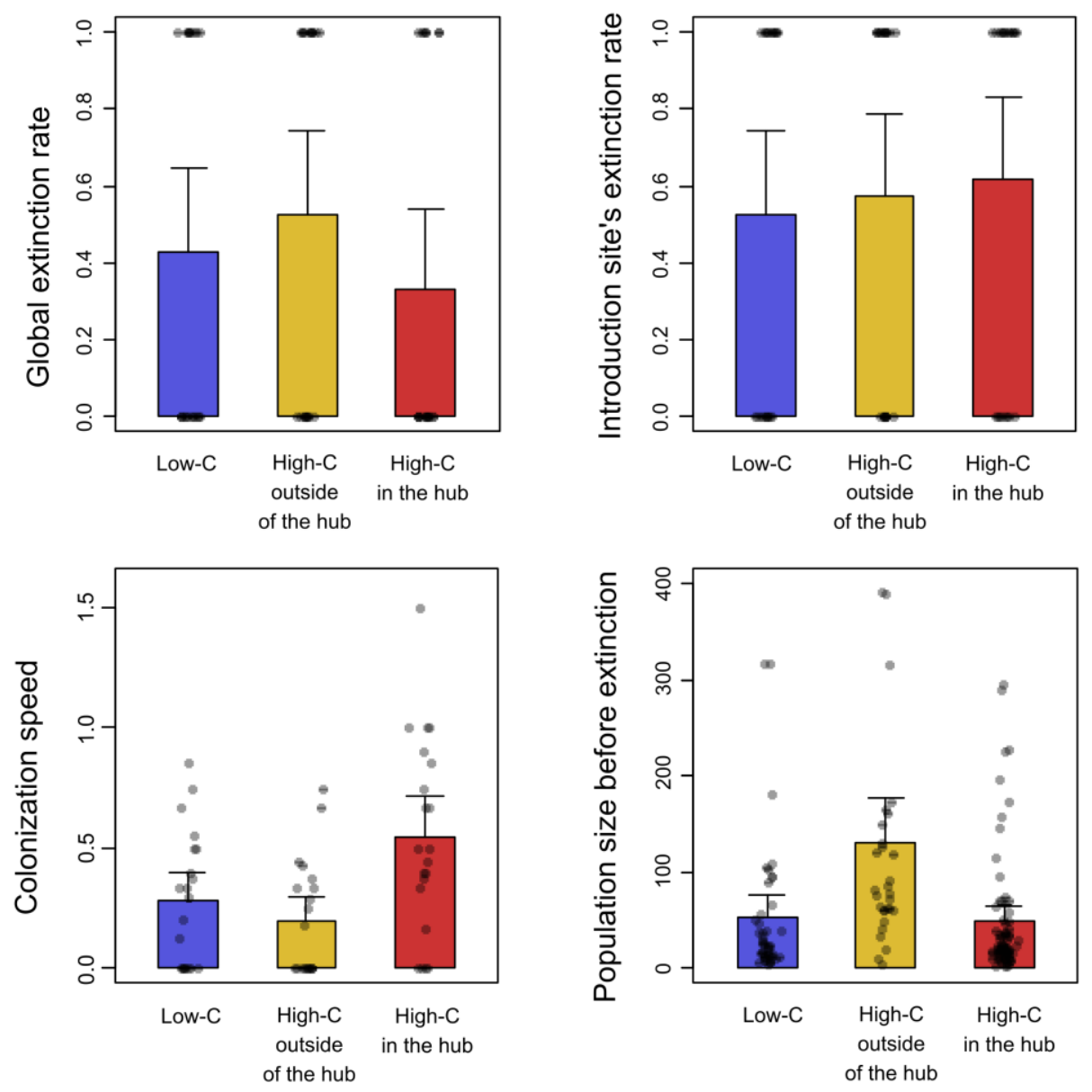

2 Figure 2: Mean experimental values for global extinction rates (A), the introduction site's

3 extinction rates (B), colonization speeds (C) and populations size before their extinction (D),

4 for the experimental introductions in low-C landscapes (blue), high-C landscapes outside of the

5 hub (yellow), and high-C landscapes in the hub (red). The error bars represent 2 times the

6 standard deviation from the mean value and the dots represent the raw values 


\section{$1 \quad$ Tables}

2 Table 1: Differences in AIC between the M-model $\left(\Delta_{C}\right)$ or the C-model $\left(\Delta_{M}\right)$ and the CM-model

3 for the simulation results. All the values greater than 10 (indicating unequivocal higher support

4 for the CM- model compared to the other one) are in bold.

5

\begin{tabular}{lllll} 
Response variable & Allee effect & Intro. site & $\Delta_{C}$ & $\Delta_{M}$ \\
\hline Global extinction rate & $a=0$ & In the hub & 2.02 & -1.49 \\
& $a=0$ & Outside & -1.99 & 0.79 \\
& $a=2.5$ & In the hub & $\mathbf{6 0 2 . 3 7}$ & -0.66 \\
Introduction site extinction rate & $a=2.5$ & Outside & -1.82 & -0.95 \\
& $a=0$ & In the hub & $\mathbf{1 7 3 . 4 5}$ & -0.11 \\
& $a=0$ & Outside & -1.31 & 0.38 \\
Colonization speed & $a=2.5$ & In the hub & $\mathbf{1 8 0 6 . 5 0}$ & 9.70 \\
& $a=2.5$ & Outside & -0.77 & -1.95 \\
& $a=0$ & In the hub & $\mathbf{3 2 5 0 . 4 5}$ & $\mathbf{8 6 5 . 1 5}$ \\
& $a=0$ & Outside & $\mathbf{1 3 0 . 5 0}$ & $\mathbf{6 4 8 . 8 0}$ \\
& $a=2.5$ & In the hub & $\mathbf{1 5 7 8 . 8 4}$ & $\mathbf{2 9 . 2 0}$ \\
& $a=2.5$ & Outside & -1.97 & -0.58
\end{tabular}

